Aus dem Medizingeschichtlichen Institut

(Direktor: Prof. Dr. E. H. Ackenknechт) der Universität Zürich

\title{
Die Historisch-Geographische Pathologie von August Hirsch
}

\section{Ein Beitrag aus dem 19.Jahrhundert zum Gestaltwandel der Krankheiten \\ Von Eugen Beck}

Krankhafte Fehlfunktionen oder Reaktionen des menschlichen Organismus manifestieren sich nach außen durch bestimmte Anzeichen oder Symptome. Die dahinterstehenden Ursachen sind unendlich an Zahl und Vielfalt, die Symptome vergleichsweise monoton und arm an Ausdrucksmöglichkeit. Um den Weg bis zur auslösenden causa zurückverfolgen zu können, müssen die Symptome in ihrem Auftreten und Zusammentreffen richtig gedeutet werden. Die Krankheitseinheit bleibt ein ständig sich veränderndes Gebilde, bis die unmittelbare Relation Ursache-Wirkung durch sie umschrieben wird. Eine erste Form des Gestaltwandels der Krankheiten bezieht sich also direkt auf den jeweiligen ärztlichen Horizont.

Ärztliches Handeln erfolgt meist schon lange vor dem Erkennen. Der Ablauf der zu erwartenden Vorgänge soll wenn möglich aufgehalten und zum Besseren gewendet werden. Gerade wegen der Monotonie der sichtbar werdenden Krankheitszeichen basiert das Handeln, die Therapie, viel mehr auf Erfahrung als auf Wissen. Das eigentliche Wirken kann aber erst dort erfolgen, wo es die causa selbst erreicht und beeinflußt. Durch Elimination, Abschwächung oder auch nur vorübergehende Beeinflussung der causa entsteht eine zweite Form des Gestaltwandels: die therapeutisch bedingte und in letzter Zeit häufig überschätzte Veränderung oder erfolgreiche Bekämpfung früher überwiegender Krankheitsformen.

Eine dritte Form des Gestaltwandels beruht auf Veränderungen der causa selbst. Diese Form wird häufig als «spontaner Gestaltwandel» bezeichnet, wobei «spontan» ganz einfach heißt, daß wir den Vorgang nicht zu deuten wissen.

Jede Form des Gestaltwandels kann nur im Verlauf der Zeit, also geschichtlich, erfaßt werden. Aus der Erkenntnis dieser Vorgänge entsteht die «Geschichte der Krankheiten», die «Historische Pathologie». August 
HrRsch erfaßte diese Denkweise konsequent vor mehr als hundert Jahren in seinem Handbuch der Historisch-Geographischen Pathologie.

Aron Simon Hirsch, später auf den Namen August getauft, wurde 1817 in Danzig geboren. Auf Wunsch seines Vaters begann er eine Handelslehre, wandte sich aber dann dem Studium der Medizin zu, welches er im Jahre 1843 mit einer Dissertation über den «Croup» abschloß. Zunächst war er als praktischer Arzt und Armenarzt in Elbing und Danzig tätig. Vor der Naturforschenden Gesellschaft Danzigs trat er mit ersten Arbeiten über das historisch-geographische Auftreten von Krankheiten an die Öffentlichkeit ${ }^{1}$. SchöNLEIN regte ihn zu einer zusammenfassenden Darstellung seiner umfangreichen Untersuchungen in einem Handbuch an ${ }^{2}$. Dieses erschien 1859 in erster Auflage im Anschluß an das Handbuch für spezielle Pathologie und Therapie von VIRchow. Nicht weniger als 15000 literarhistorische Notizen sind in zwei Bänden kritisch verarbeitet ${ }^{1}$. Hirsch wurde durch diese immense Arbeit schlagartig in weiten Kreisen bekannt und im Jahre 1863 durch seine Berufung zum ordentlichen Professor für Geschichte der Medizin nach Berlin auch anerkannt ${ }^{1-5}$. Neben der Herausgebertätigkeit an Schmidt's Jahrbüchern seien aus den folgenden Jahren Veröffentlichungen wie die Geschichte der Medizinischen Wissenschaften in Deutschland, Geschichte der Augenheilkunde, Monographien über Meningitis cerebrospinalis und Cholera, sowie die völlig überarbeitete Neuauflage seines Handbuchs der Historisch-Geographischen Pathologie, die auch in englischer Übersetzung erschien, genannt. Seine Tätigkeit als akademischer Lehrer dehnte er auch auf die Gebiete der Speziellen Pathologie und der Hygiene aus. Die Herausgabe zahlreicher in Vorbereitung befindlicher Arbeiten wurde durch seinen Tod im Jahre 1894 vereitelt.

Der Begriff der «Historisch-Geographischen Pathologie» entspricht nicht dem der «Epidemiologie». Hirsch schließt in seinen Untersuchungen nicht nur die «akuten Infektionskrankheiten», sondern auch die «chronischkonstitutionellen » Krankheiten ein. Zu den letzteren gehören Krankheits-

1 Allgemeine Deutsche Biographie, Band 50, S. 361.

2 J. PAgel, August Hirsch $\dagger$, Dtsch. Med. Wschr. 1894, 119.

${ }^{3}$ R. Virchow, August Hirsch $\dagger$, Berliner Klin. Wschr. 31 (1894) 129.

${ }^{4}$ Brief der Medizinischen Fakultät Berlin an Staatsminister H. v. MüHLER vom 11. März 1863. Ehemals Preußisches Geheimes Staatsarchiv, heute Deutsches Zentralarchiv, Abt. Merseburg (Mikrofilm).

${ }^{5}$ Posner, Die Professur für Geschichte der Medizin an der Berliner Universität, Allg. Med. Centralztg. 28 (1863) 32. 
bilder, die inzwischen als Avitaminosen, Stoffwechselstörungen oder auch Infektionskrankheiten erkannt wurden. Wir beschränken uns in dieser Arbeit auf das Gebiet der Infektionskrankheiten.

Hirsch glaubte vorerst, mit der Begründung einer modernen «Geographischen Medizin» eine neue, fruchtbringende wissenschaftliche Methode geschaffen zu haben. Tatsächlich gelang es ihm, auf Grund seiner kritischen Zusammenstellungen von Tatsachenmaterial zahlreiche der haltlosen Theorien der vorbakteriologischen Ära zu widerlegen. Er leistete auf diese Weise einen nicht zu unterschätzenden Beitrag zur Ausweitung des ärztlichen Horizonts seiner Zeit, indem er keine Mühe scheute, auch entlegenste Quellen zu verarbeiten. Leider vermochte er seine Schlüsse, die sich in großer Zahl als richtig erweisen sollten, nicht zu beweisen. Die Beweise erfolgten teilweise noch zu seinen Lebzeiten durch die Funde der Bakteriologen, deren Resultate er durchaus anerkannte, jedoch nicht vorbehaltlos akzeptierte. Diese reservierte Haltung brachte ihm den Ruf eines unzeitgemäßen Konservatismus ein. Abgesehen von der Verwendung seiner Zusammenstellungen für historische Angaben wurde er nach seinem Ableben kaum mehr zitiert. Erst in neuester Zeit hat die Geomedizin wieder an Bedeutung gewonnen im Zusammenhang welthygienischer Maßnahmen während des letzten Weltkrieges und im Rahmen der Weltgesundheitsorganisation.

Eine Geschichte der «Geographischen Medizin» läßt sich bis in ihre Anfänge zurückverfolgen. Geographische Medizin umfaßt nicht einfach die Beschreibung kurioser Krankheiten in fernen Ländern, sondern will als eigener Zweig der medizinischen Wissenschaft aus den Beziehungen der Krankheiten zu geographischen, topographischen und klimatischen Gegebenheiten Rückschlüsse auf Ätiologie und Pathogenese ziehen. Wenn wir von dem Buch Von der Luft, den Wassern und den Gegenden von Hippokrates absehen, so stammen wohl die ersten Versuche, eine umfassendere Geomedizin zu schaffen, erst vom Ende des 17. und Anfang des 18. Jahrhunderts. Friedrich Hoffmann ${ }^{6}$ (1660-1742) und Friedrich Carthe user ${ }^{7}$ (17041777) versuchten, einzelne für bestimmte Regionen typische Krankheiten zu beschreiben. Leonhard Ludwig Finke ${ }^{8}$ stellte in Anlehnung an das

${ }^{6}$ F. Hoffmann, A Dissertation on Endemial Diseases; or Those Disorders Which Arise from Particular Climates, Situations, and Methods of Living, London 1746.

7 F. Cartheuser, Descriptio I-II de morbis endemiis, Frankfurt an der Oder 1771.

${ }^{8}$ L. L. Finke, Versuch einer allgemeinen medicinisch-praktischen Geographie, worin der historische Theil der einheimischen Völker- und Staaten-Arzneykunde vorgetragen wird, 3 vol., Leipzig 1792-1795. 
System einer medizinischen Polizey von J.P. Frank eine «allgemein medicinisch-practische Geographie » auf (1792-1795). Er wollte den Völkern die konkreten Gefahren zeigen, denen sie sich aussetzten, wenn sie diese oder jene Existenzgrundlagen der Krankheiten bestehen ließen. Friedrich SchnurRer ${ }^{9}$ veröffentlichte 1813 eine Geographische Nosologie. Der «Nosologist » darf die Krankheiten nicht aus irgendwelchen speziellen Manifestationen heraus deuten, sondern er muß bis zu den "Grundgesetzen » vordringen und die Geschichte der Krankheiten als Funktion von Klima, Land und Rasse mit erfassen ${ }^{10}$. Wenig ernst zu nehmen ist die 1853 veröffentlichte Medicinische Geographie von C. F. Fuchs ${ }^{11}$. Der Autor teilt die Welt großzügig in drei Krankheitszonen ein: die katarrhalische, die enteromesenterische und die dysenterische.

Der bedeutendste Autor auf diesem Gebiet vor Hirsch ist wohl A. MüHRY ${ }^{12}$, der 1856 sein Werk Die geographischen Verhältnisse der Krankheiten, oder Grundzüge der Nosogeographie veröffentlichte. Die eigentlichen Gesetze, welche die Verteilung der Krankheiten auf der Erdoberfläche bestimmen, müßten nach ihm durch eine Untersuchung vom geographischen Standpunkt auf Grund typischer Übereinstimmungen zum Vorschein kommen ${ }^{13}$.

Nach August Hirsch ist «das menschliche Leben - in concreto - der Ausdruck des Prozesses, welcher in einem lebensfähigen Organismus durch die Summe aller auf ihn einwirkenden, äußeren Momente hervorgerufen und unterhalten wird, dessen Form somit von der Art des Organismus und dem Verhalten der ihn umgebenden Außenwelt abhängig erscheint». ${ }^{14}$ Die historisch-geographische Untersuchungsmethode hat also auch Faktoren wie Jahreszeit, Alter, Rasse und soziale Bedingungen zu berücksichtigen. Hirsch ist einem Versuch gegenüber, aus der geographischen Medizin ein

${ }^{9}$ F.S. Schnurner, Geographische Nosologie oder die Lehre von den Veränderungen der Krankheiten in den verschiedenen Gegenden der Erde, in Verbindung mit physischer Geographie und Naturgeschichte der Menschen, Stuttgart 1813.

10 A. Barkhuus, Medical Geographies, Ciba Symposia 10 (1945) 1997.

11 C. F. Fuchs, Medicinische Geographie, Berlin 1853.

12 A. MÜHRY, Die geographischen Verhältnisse der Krankheiten oder Grundzüge der NosoGeographie, Leipzig/Heidelberg 1856.

${ }^{13}$ A. Hirsch, Über den Wert und die wissenschaftliche Bedeutung der geographischen und historischen Pathologie, Münchn. Med.Wschr. 1856, 302, und Übersicht der Leistungen im Gebiete der medizinischen und geographischen Pathologie während der letzten Jahre, Schmidt's Jb. 95 (1857) 237.

${ }^{14}$ A. Hirsch, Handbuch der historisch-geographischen Pathologie, Band I, S. 1, Erlangen $1859 / 60$. 
eigenes methodisches Gebäude zu schaffen, eher zurückhaltender als seine Vorgänger. Erst wenn alle speziellen Fragen beantwortet wären, «dürfte es Zeit sein, zu allgemeinen Abstraktionen zu schreiten, mit philosophischem Geiste die Lücken auszufüllen, und ein Gebäude der allgemeinen medizinischen Geographie aufzubauen ».15

Die Grundkonzeption der Methodik läßt sich mit Hirsch wie folgt zusammenfassen :

1. größtmögliche Vollständigkeit der verwertbaren Quellen,

2. Berücksichtigung aller Krankheitsformen, welche in medizinischgeographischer Hinsicht von Interesse sind,

3. vorsichtige Handhabung der Statistik, da die aus Krankenhäusern stammenden, einzig brauchbaren Angaben nicht zu allgemeinen geographischen und epidemiologischen Schlüssen führen können,

4. möglichst vollständiger Darstellung der geographischen Verbreitung einer Krankheit sowie deren wichtigsten historischen Daten, räumlicher Antagonismus oder Koinzidenz usw., und

5. Verzicht auf die Darstellung einer speziellen Krankheitslehre, außer bei selteneren oder nach seiner Ansicht zu wenig berücksichtigten Krankheitsformen. Ebenso verzichtet der Autor auf eigene experimentelle Forschung ${ }^{16}$.

Dagegen hält er mit seinem Urteil dem gegenüber, was er für richtig oder falsch hält, immer erst nach strengster Prüfung durch seine historischgeographischen Untersuchungen, nicht zurück.

Hirsch war durchaus imstande, mit seiner Methodik alle Formen des Gestaltwandels der Krankheiten zu erfassen. Er braucht selber immer wieder den Ausdruck « Geschichte der Krankheit », sieht also in einer Krankheit etwas Lebendiges, Wandelbares. Damit stand er in krassem Gegensatz zu den Systematikern seiner Zeit (z. B. FARR ${ }^{17}$ ), die eine Systematik der Krankheiten aufzustellen versuchten, wie LINNÉ es auf dem Gebiet der Botanik gezeigt hatte. Das Anliegen Hirschs, mittels der historisch-geographischen

15 Übersicht der Leistungen im Gebiete der medizinischen Geographie, Schmidt's Jb. 78 (1853) 355.

${ }^{16}$ A. Hirsch, Handbuch der historisch-geographischen Pathologie, Band I, S. 3 f., Erlangen $1859 / 60$.

17 W. FARR, Annual Reports of the Registrar General of Births, Deaths and Marriages in England, zit. nach Wien.Med.Wschr. 1856, 304. 
Methode auch ätiologische Forschung zu treiben, erhielt das Gehör der damaligen medizinischen Welt nur in beschränktem Maß. Selbst die verschiedenen Rezensionen, die sich über sein Handbuch sehr positiv äußern, sehen in dem Werk wohl eine Möglichkeit zur Klärung vieler Begriffe, nicht aber eine Grundlage für weitere Forschung ${ }^{18-21}$. Wenn wir aber die Schlußfolgerungen einzelner Kapitel im Handbuch, vor allem über die Infektionskrankheiten, betrachten, so braucht es beinahe nur noch den Blick ins Mikroskop mit seinen Folgen für Prophylaxe und Therapie, und wir haben modernste Medizin vor uns.

Diese Behauptung sei an drei Beispielen exemplifiziert: Masern, Scharlach und Diphtherie. Alle drei Krankheiten erlebten teils vor, teils nach Hirsch wesentliche Wandlungen. Die moderne Konzeption ${ }^{22-24}$ wird jeweils derjenigen von Hirsch vorangestellt.

\section{Masern}

Das Masernexanthem soll bereits erkennbar in arabischen Schriften des 10. Jahrhunderts beschrieben worden sein. Der Ausdruck «morbilli», kleine Krankheiten, stammt von der Anschauung, daß es sich um eine besonders milde Form der Blattern handle. Das Krankheitsbild als pathogenetische Einheit wurde vermutlich zuerst von Sydenham abgegrenzt. Infektionsversuche mit blutgetränkten Wattenbäuschchen auf die inzidierte Haut wurden 1758 durch HoмE ${ }^{25}$ erwähnt. Die Übertragbarkeit wurde 1905 durch Heктоen ${ }^{26}$ an Freiwilligen bewiesen. Seit 1918 ist der Schutz vor der

18 Thierfelder sen., Rezension über A. Hirschs Handbuch der historisch-geographischen Pathologie, Band I, Schmidt's Jb. 103 (1859) 276.

19 Thierfelder sen., id. Band II, Schmidt's Jb. 108 (1860) 362.

${ }^{20}$ Wernich, Rezension über A. Hirschs Handbuch der historisch-geographischen Pathologie, zweite, neubearbeitete Auflage, Band I, Schmidt's Jb. 191 (1881) 306.

21 Wernich, id. Band II, Schmidt's Jb. 198 (1883) 299.

${ }^{22}$ R. L. CeciL und R. F. Loeb, A Textbook of Medicine, Philadelphia/London 1959.

${ }^{23}$ A. Grumbach und W. Kıкuth, Die Infektionskrankheiten des Menschen und ihre Erreger, Stuttgart 1958.

${ }^{24}$ K. KöHN und H. JANSEN, Gestaltwandel klassischer Krankheitsbilder. Eine kritische Studie zur therapeutisch bedingten Pathomorphose aus der Sicht des pathologischen Anatomen, Berlin 1957.

${ }^{25}$ F. Home, Principia Medicinae, Edinborough 1758.

${ }^{26}$ Hektoen, J. Inf. Dis. 2 (1905) 238. 
Krankheit mit Rekonvaleszentenserum bekannt ${ }^{27}$. Die Züchtung des Masernvirus gelang in letzter Zeit ebenfalls ${ }^{28,29}$. Der Kontagionsindex der Krankheit ist außerordentlich hoch. Im Jahre 1951 trat in Grönland die erste bekannte Masernepidemie auf, wobei $99,9 \%$ der eingeborenen Bevölkerung erkrankten ${ }^{30}$. Die Prognose hängt weitgehend vom Alter der Patienten und vom Auftreten von Komplikationen wie Encephalitis, Otitis media und Bronchopneumonie, meist durch Sekundärinfektion bedingt, $a b$. Die Sterblichkeit ist in Stadtgebieten größer als auf dem Lande. Sie nahm in den letzten Jahren deutlich ab, einerseits durch eine bessere Beherrschung der Sekundärinfektionen, anderseits durch eine Vermehrung von «mitis»Fällen. Die Ursache dieser Veränderungen ist unbekannt ${ }^{31}$.

August Hirsch bemerkt, daß der typische Masernausschlag von RHAzes als Hhasbah beschrieben, von Avicenna bereits mit den Blattern verwechselt wurde. Sydenham erwähnt er nicht.

Die Masern sind über die ganze Welt verbreitet, Epidemien an entlegenen Punkten jedoch sehr selten. Nachgewiesene Masernepidemien traten beispielsweise auf den Faröer-Inseln und auf Island nur in den Jahren 1644, 1694 und 1846 auf. Der Weg der Einschleppung konnte verfolgt werden. Klimatische und Bodenverhältnisse beeinflussen die Krankheit nicht, ebensowenig die Rassenzugehörigkeit. Die statistische Auswertung von 309 Epidemien zeigt ein häufigeres Auftreten in den Sommermonaten, doch können die Witterungsverhältnisse nicht als direkte Ursache angesehen werden. Auffällig ist eine Fluktuation zwischen Epidemien beinahe pandemischen Charakters und Zeiten, in welchen die Krankheit nur sporadisch auftritt.

Die Schleimhautveränderungen des Mundes betrachtet Hirsch als zuverlässigstes Symptom (37 Jahre vor KopLIK!), während das Exanthem bei den farbigen Rassen nur schwer zu erfassen sei. Ebenso pathognomonisch sind die katarrhalischen Begleiterscheinungen. In tropischen Gegenden werden Begleit-Enterokolitiden gehäuft beschrieben. Die Ansicht, daß heißes

${ }^{27}$ Ch. Nicolle und E. Conseir, Pouvoir préventif du sérum d'un malade convalescent de rougeale, Bull.Soc. Méd.Hôp. Paris 42 (1918) 336.

${ }^{28}$ H. Plotz, Culture in vitro du virus de la rougeole, Bull.Acad.Méd. 119 (1938) 598.

${ }^{29}$ J. F. Enders und T. C. Peebles, Propagation in Tissue Culture of Cytopathogenic Agents from Patients with Measles, Proc. Soc.Exper. Biol. Med. 86 (1954) 277.

${ }^{30}$ P. E. Christensen et al., An Epidemic of Measles in Southern Greenland, 1951. Measles in Virgin Soil, Acta Med. Scand. 144 (1953) 430.

${ }^{31}$ E. Glanzmann, Masern, Handbuch der Inneren Medizin, 4.Auflage, Berlin/Göttingen/ Heidelberg 1952. 
Klima zu Ruhr, kühle Witterung zu Affektionen des Respirationstraktes prädisponieren, versucht Hirsch durch Beispiele zu widerlegen: Während der Sommerepidemien der Jahre 1827 und 1837 in Deutschland wurde der Croup als Komplikation besonders häufig beobachtet. Ruhr bestimmte den Verlauf zahlreicher Frühjahrsepidemien in Deutschland und in den USA.

In einer scharfen Polemik greift Hirsch den Geomediziner Fuchs an, der behauptet, es bestehe ein Zusammenhang zwischen geographischen Gegebenheiten und der Bösartigkeit der Komplikationen ${ }^{32}$. Nach Hirsch ist der Charakter einer Epidemie als solcher festgelegt. Das Verhältnis der gutartigen zu den bösartigen Epidemien gibt er gleichmäßig über alle Jahreszeiten mit $4: 1$ bis 5,6:1 an. Gegenüber seiner Zeit (1859) seien früher die bösartigen Epidemien häufiger gewesen, was er aber in vielen Fällen falschen Maßnahmen der Krankheit gegenüber zuschreibt. Alle diese Angaben stammen aus der ersten Auflage des Handbuchs der Historisch-Geographischen Pathologie. Für die zweite Auflage von 1881 schrieb Hirsch das MasernKapitel völlig neu. Insbesondere wirft er die Frage nach dem «Wie?» des jahreszeitlichen Einflusses deutlicher auf. Die geographisch bedingte Prävalenz gewisser Komplikationen bringt er mit einem vorbestehenden «locus minoris resistentiae» in Verbindung. Anscheinend ließ er sich inzwischen doch von der Tatsache überzeugen, daß Darmaffektionen als Masernkomplikation in den Tropen gehäuft auftreten.

Die Bösartigkeit gewisser Epidemien erklärt Hirsch noch immer vorwiegend mit falschen diätetischen und hygienischen Maßnahmen. Die Epidemien des 18. Jahrhunderts und früher möchte er nur mit Vorsicht beurteilen, da Verwechslungen mit dem Scharlach noch allzu häufig vorkamen. Als Ursache gibt er in Übereinstimmung mit KLEBS und SAlisburY ${ }^{33}$ ein organisches «Gift» an, das nur in einer geringen Umgebung des Kranken übertragend wirken können. Die Krankheit dauere nur solange, als das Gift « einen zu seiner Reproduction geeigneten Boden in Individuen findet, welche für dasselbe empfänglich sind.» ${ }^{34}$

Glanzmann stellt in der letzten Auflage des Handbuchs der Inneren Medizin fest, daß «mitis»-Infektionen zugenommen hätten ${ }^{35}$. Hirsch hält die Masern-Krankheit an sich für eine der am günstigsten verlaufenden

32 Vgl. 15, S. 356.

${ }^{33}$ Zitiert nach A. HrRSCH, Handbuch der historisch-geographischen Pathologie, Erlangen 1881, S. 121.

34 a.a. O., S. 122.

${ }^{35} \mathrm{Vgl} .31$, S. 105. 
akuten Infektionskrankheiten, deren Ausgang nur durch unzweckmäßiges Verhalten in Frage gestellt würde, erwähnt aber doch in der zweiten Auflage seines Handbuchs den Ausdruck der «constitutio epidemica». Beide Autoren kommen um die Tatsache nicht herum, daß die Krankheit «spontane» Wandlungen zeigt.

\section{Scharlach}

Die «febris scarlatina» wurde erstmals von Sydenham beschrieben anhand der Londoner Epidemien einer Krankheit, die zunächst einen durchaus benignen Verlauf nahm, dann aber an Bösartigkeit der Pest nicht nachstand. Bretonneau hielt Scharlach für eine leichte Krankheit. Nach ihm wurden im 19. Jahrhundert ausgesprochen bösartige Epidemien beschrieben. Im 20. Jahrhundert verlor die Krankheit therapieunabhängig an Gefährlichkeit, in Ost- und Südeuropa jedoch später als in Westeuropa. Nach Einführung der Penicillintherapie beträgt die Letalität heute nur noch 0,1 bis $0,5 \%^{36}$.

August Hirsch leugnet, daß die Krankheit im Altertum oder Mittelalter beschrieben worden sei. Die Angaben aus dieser Zeit bezögen sich auf andere exanthematische Krankheiten. Die ersten eindeutigen Nachrichten über diese Krankheit stammen nach ihm aus dem Jahre 1627 von Döring aus Breslau ${ }^{37}$ und von Sennert aus Wittenberg ${ }^{38}$. Die Beobachtung der Krankheit erfolgte unabhängig, so daß zwar nicht geschlossen werden kann, daß die Krankheit vorher nicht existiert hätte, ein erstmaliges Auftreten des Scharlachs in größerem Ausmaß in diesen Jahren jedoch naheliegt. In den folgenden Jahren wurde das epidemische Auftreten einer neuen Exanthemkrankheit in Deutschland und England mehrfach beschrieben. Sydenham kommt auch nach Hirsch das Verdienst zu, das Krankheitsbild des Scharlachs erstmals eindeutig von den Masern abgegrenzt zu haben. Die «febris scarlatina » wurde in Frankreich erst 1712, in Italien 1717 und in Südamerika sogar erst 1829 beobachtet. In Europa starben in den Jahren 1848 bis 1855 zahlreiche Patienten an der Krankheit, so daß in diesen Jahren 4 bis $5 \%$ der Gesamtmortalität in England und Wales sich auf Scharlach beziehen.

36 Statistik der Bundesrepublik Deutschland, Band 127.

37 Vgl. Sennert, Epist. Cent. I. Ep. 18.

38 Sennert, ibid. Ent. II. Ep. 20 (37 und 38 zitiert nach A. HIRSCH, Handbuch der historischgeographischen Pathologie, Erlangen 1859/60, S. 235). 
In Afrika ist nach Hirsch die Krankheit sehr selten. Noch heute besteht die Ansicht, daß die schwarze Rasse gegen Scharlach weitgehend immun sei. Hirsch betont aber, daß in Südamerika die Krankheit bei allen Rassen gleich verlaufe. Jahreszeit, Klima und Witterung beeinflussen die Morbidität nicht. Im periodischen Auftreten von Pandemien verhält sich Scharlach ähnlich wie die Masern, doch folgen sich die Epidemien - dies wird in der Auflage von 1881 vermerkt - bei Scharlach in größeren Abständen als bei Masern. Wenn die Krankheit in einem Gebiet einmal aufgetreten ist, so verharrt sie dort oft jahrelang. Sporadische Fälle sind weit häufiger als bei Masern. Der Charakter der Scharlachepidemien schwankt außerordentlich stark: Die Letalität schwankt zwischen 3 und $30 \%$, wobei einige besonders schwere Epidemien in den Tropen beobachtet wurden.

Ätiologische Vermutungen über das Scharlachfieber werden von Hirsch in der zweiten Auflage seines Handbuchs angegeben, während er sich 1860 noch sehr zurückhaltend äußert. Obschon KocH bereits 1878 die pathogene Wirkung der Streptokokken im Tierversuch nachwies, so stammt die Beschreibung des «streptococcus scarlatinae» durch KLEIN ${ }^{39}$ doch erst aus dem Jahre 1887. Hirsch schließt jedenfalls eine autochthone Entstehung des Scharlachfiebers aus. Verantwortlich soll ein organisches Gift sein. Die Verbreitung der Krankheit hängt von der Reproduktion desselben und von seiner Ausscheidung durch den Patienten ab. Die Übertragung erfolgt durch die Luft in naher Umgebung der Kranken oder durch infizierte Gegenstände. Auch «healthy carriers » werden als Krankheitsvermittler erwähnt, indem sie «mit ihrem Körper oder ihren Kleidungsstücken den Träger des Krankheitsgiftes abgeben . $^{40}$ Ärzte konnten auf diese Weise ihre eigenen Patienten anstecken.

Auch bei dieser Krankheit sind wesentliche Angaben über ihr epidemiologisches Verhalten und ihren Gestaltwandel schon vor hundert Jahren festgehalten. Mögen die Vertreter der Penicillin-Ära nicht zu dem gleichen Schluß kommen wie Graves im Jahre 1843: In den Jahren 1801 bis 1804 traten in Dublin außerordentlich schwere Scharlachepidemien auf, dann änderte sich der Charakter der Krankheit schlagartig, und die Epidemien verliefen in den folgenden 27 Jahren sehr gutartig. Graves schreibt: «Was war natürlicher, als daß wir, übermütig gemacht durch unsere glücklichen Erfolge, das Verdienst uns selbst zuschrieben und die große Sterblichkeit

${ }^{39}$ E. Klein, Etiology of Scarlet Fever, Proc.Roy. Soc. Med.London 42 (1887) 158.

${ }^{40}$ A. Hinsch, Handbuch der historisch-geographischen Pathologie, Erlangen 1881, S. 139. 
in den früheren Epidemien einzig und allein auf Rechnung der damals stattfindenden irrigen Behandlung (im Geiste der Brownschen Schule) stellten. ${ }^{41}$ Die Epidemien von 1834/35 zerstörten diese Vorstellung durch ihren bösartigen Verlauf sehr schnell.

\section{Diphtherie}

Bretonneau beschrieb die Krankheit in seiner klassischen Monographie im Jahre 1826. KLEBS entdeckte den Erreger 1883. Im gleichen Jahr erschien der zweite Band des Handbuchs von Hirsch. v. BeHRING führte die Serumtherapie 1890 ein. Seit 1923 ist nach der Methode von Ramón die aktive Immunisierung des Menschen mit dem Formoltoxoid möglich geworden.

Epidemiologische Beobachtungen über Diphtherie aus einer «unvoreingenommenen » Zeit sind um so interessanter, als drei verschiedene Typen des Corynebacteriums bestehen, die sich sowohl in ihrem Wachstum als auch in ihrer pathogenetischen Wirkung unterscheiden. Je nach der Schwere des Krankheitsbildes, das sie hervorrufen, unterscheidet man «gravis», «intermedius»- und «mitis»-Formen. Die erstere scheint mehr epidemisch aufzutreten, während die letzteren vorwiegend endemisch vorkommen. Durch Bakteriophageninfektion entsteht eine Störung des Atemmechanismus der lysogenen Stämme, wobei ein Apoferment frei wird und als Toxin auf den menschlichen Organismus einwirkt ${ }^{42}$.

Auch bei diesem Krankheitsbild beobachten die modernen Autoren einen wesentlichen Gestaltwandel, der zeitlich etwas vor der Einführung der Serumtherapie v. Behrings einsetzt. Abgesehen von einer Besserung der Prognose scheinen die Fälle von Croup seltener und die Herzveränderungen häufiger geworden zu sein: Der «Würgeengel» wurde zum «Herzensbrecher $\gg .{ }^{43}$ In den Jahren nach dem Zweiten Weltkrieg traten schwere Formen in Deutschland vorübergehend wieder häufiger auf.

August Hirsch stellt fest, daß die Krankheit seit dem Altertum bekannt sei. In einer tabellarischen Zusammenstellung faßt er 138 Epidemien von 1583 bis 1860 zusammen, wobei er nur solche erwähnt, die er aus den Quellen schlüssig als Diphtherie-Epidemien erkennen konnte. Drei Krankheits-

${ }^{41}$ Graves, System of Clinical Medicine, Leipzig 1843, S. 65 (zitiert nach Hirsch, Handbuch ..., Erlangen 1881, S. 129).

42 N. B. Groman, Evidence for the Active Role of Bacteriophage in the Conversion of Nontoxigenic Corynebacterium diphtheriae to Toxin Production, J. Bact. 69 (1955) 9. ${ }^{43}$ Th. Brugsch, Ther. Gegenw. 1943, 41. 
formen ließen sich immer wieder beobachten: eine leichte Angina mit Schluckbeschwerden, die der Patient nach sechs bis zehn Tagen überwindet, eine schwerere Form, bei welcher Atemkomplikationen im Vordergrund stehen (Garotillo) und die Angina maligna mit «toxischem» Verlauf. Bei den letzteren Formen ist die Prognose schlecht. Häufig wird eine Epidemie durch eine bestimmte Verlaufsform gekennzeichnet. In den Jahren nach 1846 beobachtete Trousseau in Paris eine besonders fulminante Form, die Bretonneau noch unbekannt war, bei welcher der betroffene Patient ohne Larynxbeteiligung innert 48 Stunden starb. Hirsch spricht von einem typhoiden Charakter dieses Krankheitsbildes.

Die Diphtherie kam in der Zeit vor Hirsch selten sporadisch vor. Meist wurden ganze Länderkomplexe während Jahren epidemisch erfaßt, um dann wieder für Jahre völlig verschont zu werden. Erst nach 1820 ist eine geographisch-medizinische Untersuchung möglich: Wiederum zeigen geographische, klimatische und Witterungseinflüsse nach statistischer Auswertung der zuverlässigeren Quellen keinen nennenswerten Einfluß auf die Entstehung der Krankheit. Dagegen sollen, «wie bei allen zymotischen Krankheiten», soziale Bedingungen einen wesentlichen Einfluß ausüben. Das Vorhandensein eines «contagium», besonders in Fällen mit Hautdiphtherie, setzt Hirsch als selbstverständlich voraus. In der zweiten Auflage des Handbuchs wird ferner die Möglichkeit von Epizootien in Erwägung gezogen. Die bakteriologischen Funde erwähnt Hirsch noch nicht.

Diese wenigen Beispiele mögen illustrieren, wie wertvoll, ja unentbehrlich die Arbeiten von August Hirsch für denjenigen sind, der sich mit epidemiologischen Fragen, dem Gestaltwandel der Krankheiten in jeder Form, befassen will. Die Methodik Hirschs ließ sich einigermaßen darstellen. Was nicht zum Ausdruck zu bringen ist und die Lektüre der Schriften Hirschs oft recht mühsam gestaltet, ist die geradezu erschreckende Fülle des verwerteten literarischen Materials. Der Autor scheute keine Mühe, noch entlegenere Zeugnisse anzuführen, wenn die naheliegenden Beschreibungen eines Krankheitsbildes für eine ausreichende Erklärung der Vorgänge nicht ausreichten. Die Historisch-Geographische Pathologie und ihr Autor wurden trotzdem rasch vergessen, weil die medizinische Forschung einen andern Weg nahm, als Hirsch voraussah. Anstelle des Lehrgebäudes der Geomedizin trat das Experiment. Die Epidemien der letzten Jahre, insbesondere die Grippepandemien mit all ihren ungelösten Problemen trotz genauer Kenntnis der Erreger, brachten die Bedeutung der epidemiologischen Zusammenhänge wieder weiteren Kreisen zum Bewußtsein. 